R\&D towards the module and service structure design for the ATLAS inner tracker at the super LHC (SLHC)

This article has been downloaded from IOPscience. Please scroll down to see the full text article.

2010 JINST 5 C12056

(http://iopscience.iop.org/1748-0221/5/12/C12056)

View the table of contents for this issue, or go to the journal homepage for more

Download details:

IP Address: 137.138.124.142

The article was downloaded on 11/07/2011 at 12:48

Please note that terms and conditions apply. 


\section{$R \& D$ towards the module and service structure design for the ATLAS inner tracker at the super LHC (SLHC)}

Y. Ikegami, ${ }^{a, 1}$ G. Barbier, ${ }^{b}$ F. Cadoux, ${ }^{b}$ A. Clark, ${ }^{b}$ D. Ferrere, ${ }^{b}$ S. Gonzalez-Sevilla, ${ }^{b}$ K. Hara, ${ }^{c}$ T. Kohriki, ${ }^{a}{ }^{\text {D. La Marra, }}{ }^{b}$ G. Pelleriti, ${ }^{b}$ S. Terada, ${ }^{a}$ Y. Unno ${ }^{a}$ and M. Weber ${ }^{b}$

${ }^{a}$ KEK, High Energy Accelerator Organization, INPS,

1-1 Oho, Tsukuba, Ibaraki 305-0801, Japan

${ }^{b}$ Section de physique, Université de Genève,

24, rue Ernest Ansermet CH-1211 Genève, Switzerland

${ }^{c}$ School of Pure and Applied Sciences, University of Tsukuba,

1-1-1 Tennodai, Tsukuba, Ibaraki 305-8571, Japan

E-mail: ikegami@post.kek.jp

ABSTRACT: We have designed modules and a service structure of silicon microstrip detectors as a part of the ATLAS inner tracker for the SLHC project on the basis of a modular and replaceable concept. Six modules have been completed with common components and by similar procedures. Single module tests and four-module combined tests were performed at each site and have been compared for crosschecking. Details of the module design and electrical performance are presented. A half-module was irradiated up to $5 \times 10^{14} 1-\mathrm{MeV}$ neq $/ \mathrm{cm}^{2}$ using $24-\mathrm{GeV}$ protons at the CERN PS. Its electrical performance was investigated before and after irradiation. The design of an eight-module structure, which is insertable to and is replaceable from the overall structure, has also been reported.

KEYWORDS: Si microstrip and pad detectors; Particle tracking detectors (Solid-state detectors)

\footnotetext{
${ }^{1}$ Corresponding author.
} 


\section{Contents}

1 Introduction $\quad 1$

2 Module $r$

3 Irradiation test at CERN PS $\quad 3$

4 Combined-module tests $\quad 3$

5 Super-Module program $\quad 4$

6 Summary 5

\section{Introduction}

The SLHC [1] project is aimed at integrated luminosity of $3000 \mathrm{fb}^{-1}$ with an instantaneous luminosity of $10^{35} \mathrm{~cm}^{-2} \mathrm{~s}^{-1}$. The expected radiation fluence [1], including a safety factor of 2, is (7-11) $\times 10^{14} 1-\mathrm{MeV}$ neq $/ \mathrm{cm}^{2}$ for a short-strip $\left(\mathrm{R}>38 \mathrm{~cm}\right.$ ) region and (3-6) $\times 10^{14} 1-\mathrm{MeV}$ neq $/ \mathrm{cm}^{2}$ for a long-strip $(\mathrm{R}>85 \mathrm{~cm})$ region, where the ranges within the parentheses are the highest fluence values in the central and forward regions, respectively. The main goal of this $R \& D$ program [2] is to prove that a modular silicon-strip tracker design can satisfy the required material, mechanical, electrical, and thermal performance specifications throughout the SLHC period. The R\&D program places considerable emphasis on design aspects that minimize the effort and cost involved in development and construction, while maintaining an optimal material budget. The present ATLAS silicon strip detector (SCT) [3] has also been developed using the modular concept. Modularity allows to achieve production, quality assurance and repairs in parallel. This design can ensure sufficient z-overlapping of the modules to minimize the insensitive area.

Four and two modules with common components have been built by similar procedures at the University of Geneva and KEK, respectively. In addition, a half-module has been built and irradiated up to $5 \times 10^{14} 1-\mathrm{MeV}$ neq $/ \mathrm{cm}^{2}$ using $24-\mathrm{GeV}$ protons at the CERN PS. In this study, the gain and noise characteristics of the modules before and after irradiation were compared. Singlemodule tests, four-module combined tests, and their comparison for cross checking were carried out at each site. In this paper, the details of the module design and electrical performance are presented together with the status of an eight-module installation in a realistic support structure.

\section{Module}

Figure 1 shows a photograph of a module for the barrel SCT upgrade for the SLHC experiment. It is composed of two single-sided sensors glued back to back with a baseboard in between and 


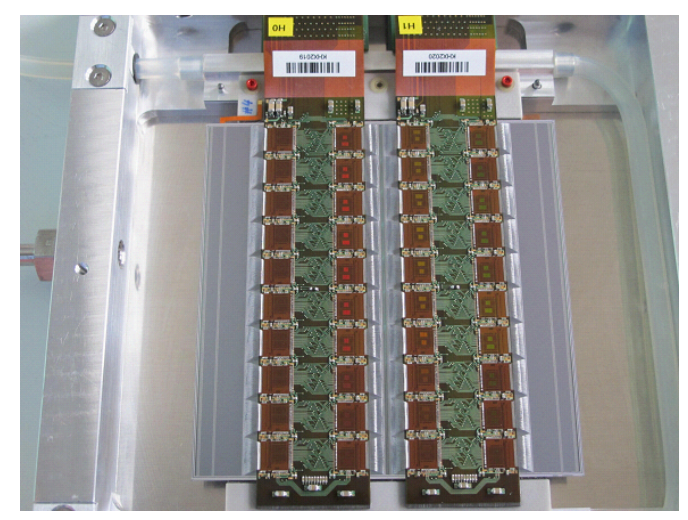

Figure 1. Module and module test box.

four separate hybrids (two on each side) bridging across the sensor area. Because the distance between the sensors, which have a stereo angle of $40 \mathrm{mrad}$, is approximately only $0.5 \mathrm{~mm}, 3 \mathrm{D}$ space points can be constructed as in a double-sided module. We have developed [4] n-in-p type strip sensors that can cope with a fluence of up to $10^{15} 1-\mathrm{MeV} \mathrm{n} \mathrm{n}_{e q} / \mathrm{cm}^{2}$. The sensor size is 97.5 $\mathrm{mm} \times 97.5 \mathrm{~mm}$, designed on a $6 / \prime$ wafer. The sensor is segmented in four parts, having a strip length of $24 \mathrm{~mm}$ as set by hit occupancy. Each segment has 1,280 strips at a 74.5- $\mu \mathrm{m}$ pitch. The $400-\mu \mathrm{m}$-thick baseboard made of thermal pyrolytic graphite (TPG) is a mechanical support, which provides excellent electrical and thermal properties. The in-plane thermal conductivity of TPG is $1450 \sim 1850 \mathrm{~W} / \mathrm{mK}$. A parylene coating technique is adopted to prevent the delamination of the TPG substrate. Four aluminium nitride (AIN) ceramic-facing plates are attached along the two sides of the substrate, which are the piers of the hybrids and have precision holes for module mounting. Each hybrid [5] has 20 front-end (FE) readout ASICs (ABCN) [3]. Each ABCN FE chip can handle 128 channels. One module has 80 readout $A B C N$ chips and thus 10,240 readout channels. The hybrid is a $136 \mathrm{~mm} \times 28 \mathrm{~mm}$ flexible $\mathrm{Cu}$ /polyimide (PI) sheet. The total thickness is $0.295 \mathrm{~mm}$ at the ASIC part and $0.165 \mathrm{~mm}$ at the cable part. The $\mathrm{Cu} / \mathrm{PI}$ sheets are prepared by an adhesive-less technology, and using button plating at through-holes. The use of button plating has resulted in a considerable weight reduction of $1.18 \mathrm{~g}$ as compared to the usual panel plating technology. The bare flexible circuit weighs only $1.91 \mathrm{~g}$. The hybrid, backed with a $400-\mu \mathrm{m}$-thick and 112-mm-wide carbon-carbon sheet, is designed to bridge over the silicon sensor to avoid any interference with the sensor surface. The carbon-carbon bridge having a high heat conductivity of $670 \mathrm{~W} / \mathrm{m} / \mathrm{K}$ transfers the generated heat to the heat sink coupled to the AlN-facing plates. The total weight (excluding electrical components) is $4.25 \mathrm{~g}$, having $0.00425 \mathrm{X}_{0}$ equivalent radiation lengths. Forty such hybrids have been successfully built. The dead-channel rate and the dead-chip rate were found to be $0.2 \%$ and $0.2 \%$, respectively, for probed ASIC chips.

Four and two modules with common components have been built by similar procedures at the University of Geneva and KEK, respectively. Uniform distributions of gain and equivalent noise charge (ENC) were observed. The average of gain and ENC were calculated to be approximately $105 \mathrm{mV} / \mathrm{fC}$ and $580 \mathrm{e}$, respectively. These results were found to be in good agreement with those anticipated from our design [3]. The module assembly, thermal performance simulation, and single module electrical tests were described in detail in ref. [6]. 

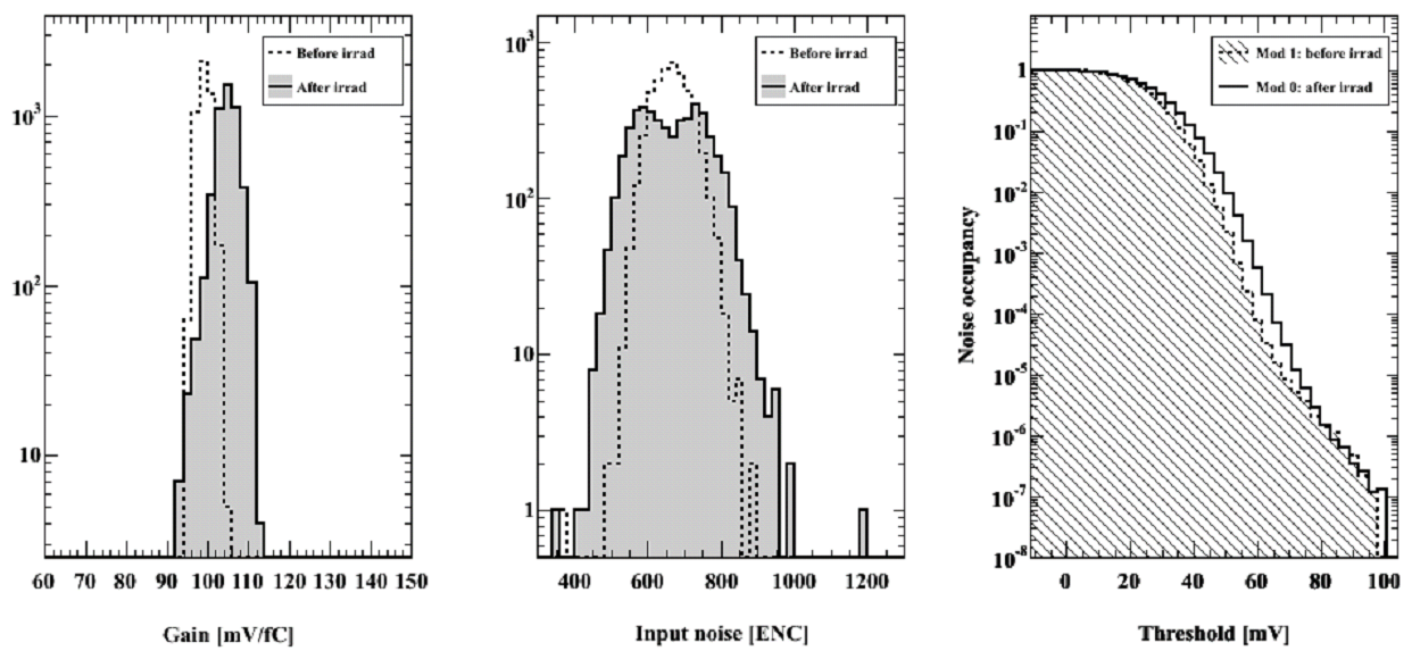

Figure 2. Distributions of gain, ENC, and NO before and after irradiation.

\section{Irradiation test at CERN PS}

Proton irradiation was performed at CERN PS in November 2009; 24-GeV protons were irradiated over a half-module, which was set on a motorized scanning table $(x, y, \theta)$. The module was inclined to the beam axis to minimize the scan area to approximately $10 \mathrm{~cm}^{2}$. The sensor was biased at $100 \mathrm{~V}$, and the chips were powered and clocked during irradiation. The temperature of the module was maintained at $-25 \mathrm{C}^{\circ}$. The hybrid temperature was approximately $+3 \mathrm{C}^{\circ}$. The fluence was estimated from the activity of the aluminium foils, which were attached to the front and rear of the test box. The measured fluence and its error were found to be $5 \times 10^{14} 1-\mathrm{MeV} \mathrm{n}_{e q} / \mathrm{cm}^{2}$ and $\pm 7 \%$, respectively.

Figure 2 shows the distributions of gain, ENC, and noise occupancy (NO). No decrease in gain was observed after irradiation. No significant degradation in terms of ENC or NO was observed after irradiation. (Because it was the first module, the DAQ software for NO was under development. Therefore, NO values before and after irradiation of two different modules were compared.) The measurement of charge collection efficiency using the binary readout ABCN chips is still under investigation.

\section{Combined-module tests}

The purpose of the combined-module tests is to test four modules placed together in a common test box to investigate the possible interference between the modules. Figure 3 shows a photograph of a four-module test box, where only three modules are installed. The first combined-module tests for 3 modules were performed at the Geneva site. Figure 4 shows the distributions of gain, ENC, and NO distributions, respectively. No significant degradation was observed between single- and combined-module tests. These tests will be continued at the Geneva and KEK sites and will be cross checked. 


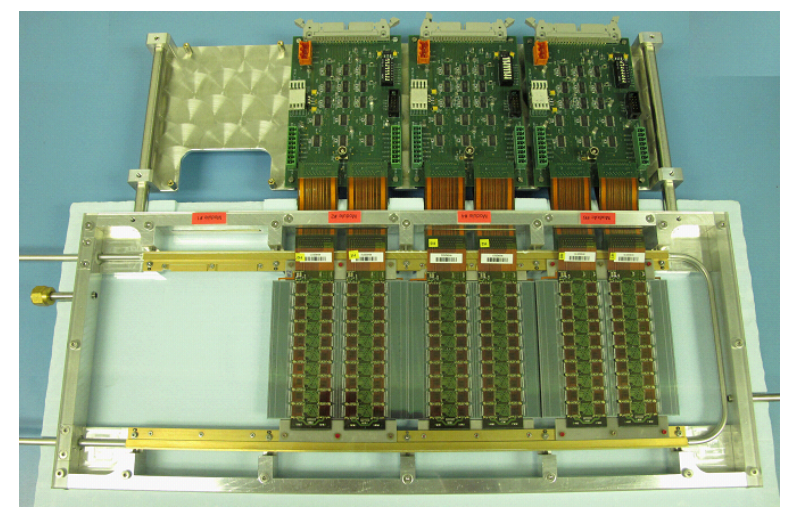

Figure 3. Four-module test box where three modules are installed.
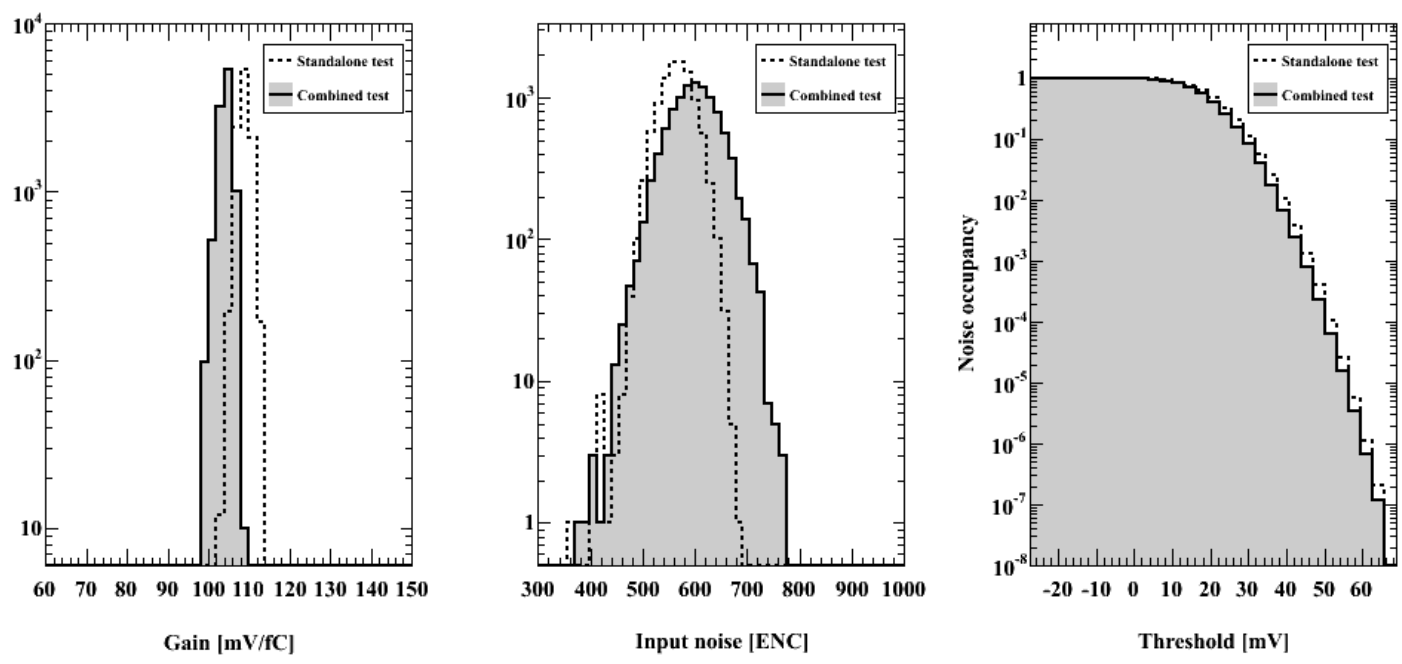

Figure 4. Distributions of gain, ENC, and NO for single- and combined-module tests.

\section{Super-Module program}

We plan to construct an assembly of eight modules placed together on a realistic support: a SuperModule. This design allows the insertion of the Super-Module from the end of the overall structure (e.g., barrel cylinder). This end insertion concept will provide a possibility of rework, allowing flexibility during integration and commissioning.

The Super-Module design is as follows. It comprises three types of bus lines: high voltage, low voltage, and data bus. The low-voltage power for the module is supplied from a DC/DC plug-in board [7], which steps down to $+2.5 \mathrm{~V}$ from $+12 \mathrm{~V}$ on the low-voltage bus line. Data communication with the modules is performed through a buffer control chip (BCC) board. The $\mathrm{BCC}$ receives signals such as clock and command signals, via the data bus line with LVDS multi drop connections. The maximum transfer rate from the BCC is $160 \mathrm{MHz}$. The end of the bus line is connected to a Super-Module board (SMB), which is an interface for an HSIO board. The Super-Module is cooled using either $\mathrm{C}_{3} \mathrm{~F}_{8}$ or $\mathrm{CO}_{2}$. 


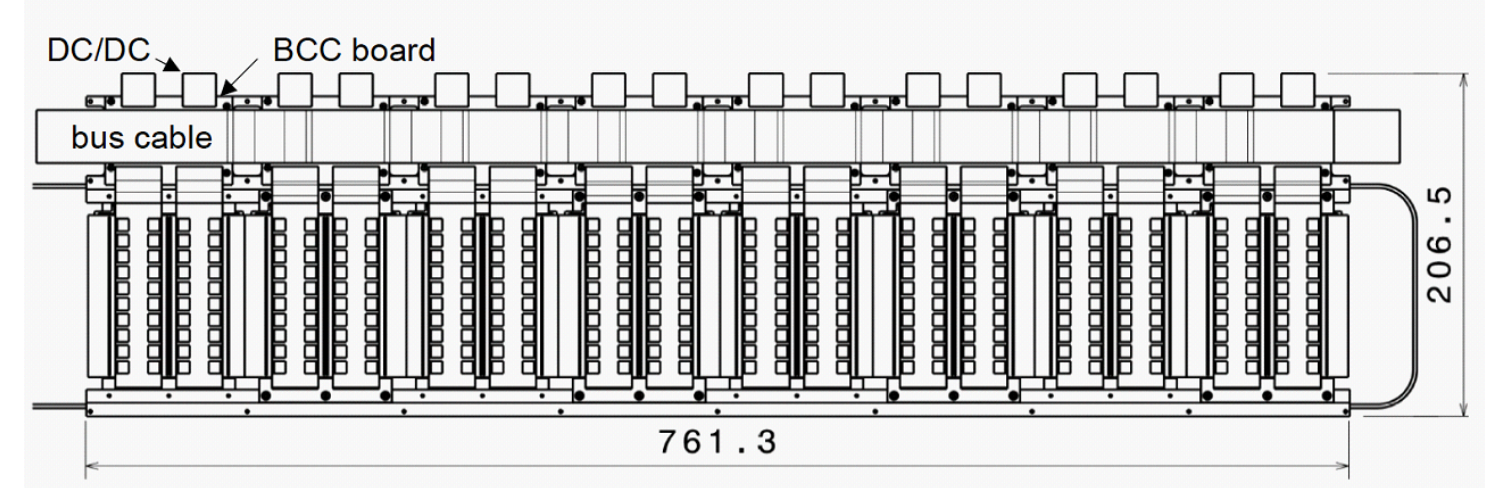

Figure 5. Super-Module program.

The design of the Super-Module has been completed, and the components will become available soon. We expect to start its testing in December 2010.

\section{Summary}

On the basis of a modular and replaceable concept, we have designed modules and a service structure of the ATLAS silicon microstrip tracker for the SLHC project.

Forty hybrids were successfully built. The dead-channel rate and dead-chip rate of the probed ASIC chips were found to be $0.2 \%$ and $0.2 \%$, respectively. Four and two modules having common components and similar building procedures have been developed by the University of Geneva and KEK, respectively. The gain and ENC were observed to be uniform across the modules, with the averages of gain and ENC being about $105 \mathrm{mV} / \mathrm{fC}$ and $580 \mathrm{e}$, respectively. These results were found to be in good agreement with the expected results.

A half-module was irradiated up to $5 \times 10^{14} 1-\mathrm{MeV} \mathrm{n}_{e q} / \mathrm{cm}^{2}$ using $24-\mathrm{GeV}$ protons at the CERN PS. No significant degradation by irradiation was found in gain, ENC, and NO.

We have also performed combined-module tests. No significant degradation in terms of gain, ENC, and NO was observed between single- and combined-module configurations.

The design of a Super-Module has been completed, and the components will become available soon. We expect to start with its testing in December 2010.

\section{Acknowledgments}

The research was partly supported by Grants-in-Aid for Scientific Research (A) (Grant No. 20244038) and on Priority Area (Grant No. 20025007) in Japan.

\section{References}

[1] J. Tseng, ATLAS Upgrade Plans for the SLHC, Nucl. Phys. B 177 (2008) 212. 
[2] M. Miñano, ATLAS Tracker Upgrade: Silicon Strip Detectors and Modules for the sLHC, 35th International Conference of High Energy Physics, PoS (ICHEP 2010) 507.

[3] ATLAS SCT collaboration, A. Abdesselam et al., The integration and engineering of the ATLAS SemiConductor Tracker, 2008 JINST 3 P10006.

[4] K. Hara et al., Development of Radiation Hard $N^{+}$-on-P Silicon Microstrip Sensors for Super LHC, IEEE Trans. Nucl. Sci. 56 (2009) 468.

[5] Y. Ikegami et al., Development of low-mass, high-density, hybrid circuit for the silicon microstrip sensors in high track density environment, PoS (RD09) 021.

[6] S. Gonzalez-Sevilla et al., A silicon strip module for the ATLAS Inner Detector upgrade in the Super LHC, to be published in the proceedings of 7th International "Hiroshima" Symposium on Development.

[7] C. Fuentes, et al., Power distribution with custom DC-DC converters for SLHC trackers, IEEE Nucl. Sci. Symp. Conf. Rec. (2009) 1300. 